\title{
APLICAÇÃO DO RESÍDUO ESPECÍFICO NA ANÁLISE DE GRUPOS DE EXPERIMENTOS $\left({ }^{1}\right)$
}

\author{
WILSON ALVES DE OLIVEIRA $\left(2^{*}\right)$; MARIA CRISTINA STOLF NOGUEIRA $\left({ }^{3}\right)$
}

\begin{abstract}
RESUMO
A análise de grupos de experimentos tem como objetivo principal produzir informações que possam ser aplicadas a uma população, seja no espaço e/ou no tempo. O objetivo deste trabalho é aplicar a técnica do resíduo específico para contornar o problema da heterocedasticidade presente nas interações dos experimentos com tratamentos, e obter as esperanças dos quadrados médios, para os efeitos considerados na análise de variância. Os dados utilizados referem-se a grupo de 11 experimentos, de adubação, no esquema fatorial $3^{3}$ com cana de açúcar, desenvolvidos em diferentes localidades do Estado de São Paulo. Os resultados revelaram que a técnica do resíduo específico é adequada quando a heterocedasticidade está presente nas interações.
\end{abstract}

Palavras-chave: interação, heterogeneidade de variâncias, contraste ortogonal, grupos de experimentos.

\section{ABSTRACT \\ APPLICATION OF SPECIFIC ERROR IN THE ANALYSIS OF GROUPS OF EXPERIMENTS}

The main aim of the analysis of groups of experiments is to produce information that can be applied to a population either in space or in time and in both. The aim of this paper is to apply the specific error technique in order to solve the heterogeneity problem of the interactions of experiments with treatments, and to obtain the expectations of the mean squares for the considered effects in the analysis of variance. The data that were used refer to a group of 11 experiments, in a $3^{3}$ factorial design with sugar cane, conducted at different places of Sao Paulo State, Brazil. The results showed that the specific error technique is adequate when the heterogeneity is present in the interactions.

Key words: interaction, heterogeneity of variances, orthogonal contrast, groups of experiments.

(1) Recebido para publicação em 8 de julho de 2005 e aceito em 9 de maio de 2007.

$\left({ }^{2}\right)$ Centro de Ciências Exatas e Tecnológicas da Universidade Estadual do Oeste do Paraná - UNIOESTE, Caixa Postal 801, 85814-110 Cascavel(PR). E-mail: waolivei@esalq.usp.br. * Autor para correspondência.

(3) Departamento de Ciências Exatas - ESALQ/USP, Caixa Postal 09, 13418-900 Piracicaba (SP). E-mail: mcsnogue@carpa.ciagri.usp.br 


\section{INTRODUÇÃO}

Experimentos agrícolas envolvendo o mesmo fator ou fatores podem ser desenvolvidos em vários locais distintos e/ou em várias ocasiões diferentes. Algumas vezes, o objetivo da pesquisa é produzir informações para uma população que se estende, no espaço e/ou no tempo.

A análise estatística apropriada para dados de grupos de experimentos vai variar com o objetivo da pesquisa. Todavia, os passos preliminares da análise tendem a ser os mesmos em todos os casos e uma aplicação, não crítica, dos métodos apropriados para análise conjunta, pode levar a conclusões errôneas.

YATes e Cochran (1938) sugerem um exame cuidadoso dos resultados experimentais antes de iniciar qualquer análise combinada, e atestam que o procedimento de análise de variância usual, apropriado para analisar os resultados de um só experimento, pode requerer modificação, devido à heterocedasticidade presente entre os diferentes experimentos e, podendo também, ocorrer a heterocedasticidade entre os componentes da interação de tratamentos com locais. Assim, sugerem a partição das somas de quadrados devido a tratamentos e da interação de tratamentos com locais em componentes ortogonais apropriados.

KeMPTHORNe (1952) considera que na análise conjunta, existem duas possíveis dificuldades para a interpretação dos resultados na análise de variância. A primeira dificuldade é que a variância residual $\left(\sigma^{2}\right)$ não é constante entre os experimentos, e a segunda, é que o componente de variância referente à interação de tratamentos com locais $\left(\sigma_{t e}^{2}\right)$ depende da combinação de tratamentos e locais.

Cochran e Cox (1957) fazem duas críticas sobre a análise preliminar. A primeira crítica, é que alguns componentes da soma de quadrados da interação de tratamentos com experimentos, podem ser maiores que outros, ou seja, a variância da interação não é homogênea. Nesse caso, o teste F para tratamentos tendo como denominador a interação de tratamentos com experimentos é viciado, no sentido de que o valor de F, obtido nas tabelas é muito baixo, isto é, obtêmse muitos resultados significativos. Segundo os autores, um método para contornar essa dificuldade é decompor a soma de quadrados de tratamentos em um conjunto de componentes ortogonais, que fornecerá toda ou a maioria das informações de interesse. A soma de quadrados das interações é decomposta do mesmo modo, obtendo-se um resíduo específico, para testar cada um dos componentes de tratamentos. Uma segunda crítica é que as variâncias dos erros experimentais não são as mesmas em todos os experimentos, podendo essa variação invalidar o teste $\mathrm{F}$ para interações tendo como denominador o Resíduo Médio.

Em Gomes (1990), constata-se aplicação do método da decomposição da interação de tratamentos com locais, em análise de grupos de experimentos de adubação de algodão. $\mathrm{O}$ autor, ao fazer a análise conjunta dos diversos experimentos, considera individualmente os graus de liberdade relativos aos contrastes de tratamentos e estima os contrastes em cada local, obtendo assim, o quadrado médio apropriado para testar cada um dos contrastes. Alerta, porém, para o fato que esse método tem a desvantagem de reduzir excessivamente o número de graus de liberdade para os testes, recomendando seu uso somente quando for grande o número de locais.

Nogueira (2004) realizou revisão sobre definições e conceitos envolvendo contrastes, buscando justificar que a técnica de contrastes ortogonais, com um número de graus de liberdade, é simples e bastante eficiente na análise de dados experimentais, tais como: obtenção dos efeitos principais, de efeito de interação, de efeitos aninhados, nas comparações entre grupos de médias e na obtenção do resíduo específico.

Neste trabalho, é aplicada a técnica do resíduo específico para contornar o problema da heterocedasticidade das interações e, são demonstradas as esperanças dos quadrados médios para os efeitos considerados na análise de variância.

\section{MATERIAL E MÉTODOS}

\subsection{Material}

Os dados analisados referem-se a rendimentos de cana-de-açúcar, em $\mathrm{t} \mathrm{ha}^{-1}$, obtidos de grupo de 11 experimentos, de adubação $\mathrm{N} \times \mathrm{P} \times \mathrm{K}$, instalados em terra roxa legítima, em diferentes localidades do Estado de São Paulo, retirado de Malavolta et al (1963).

O nitrogênio foi utilizado nas doses de 0,60 e $120 \mathrm{~kg} \mathrm{ha}^{-1}$, sendo $1 / 3$ no plantio, $1 / 3$ em cobertura, aos 3 meses, e $1 / 3$ aos 6 meses, quando ainda era usado como adubo nitrogenado o salitre-do-chile. $\mathrm{O}$ fósforo, usado nas doses de 0,75 e $150 \mathrm{~kg} \mathrm{ha}^{-1} \mathrm{de}_{2} \mathrm{O}_{5}$, foi fornecido todo no plantio, metade como superfosfato simples e metade como hiperfosfato (atualmente não utilizado). O potássio, aplicado sob a forma de cloreto, todo no plantio, teve as doses de 0, 75 e 150 kg.ha ${ }^{-1}$ de $\mathrm{K}_{2} \mathrm{O}$. 
Os experimentos foram instalados no esquema fatorial $3^{3}$, com confundimento de 2 graus de liberdade da interação tripla $\mathrm{N} \times \mathrm{P} \times \mathrm{K}$, de acordo com o grupo W (YATES, 1937), gerando três blocos envolvendo nove tratamentos para cada bloco, com uma repetição. Neste caso, segundo BANZATto e KRONKA (1989), entre outros, a parte não confundida da interação tripla, com seis números de graus de liberdade, funciona como resíduo. Visando obter resultados mais amplos e detalhados, desdobraramse as interações duplas, isolando-se apenas a interação entre os componentes lineares e juntandose os outros três componentes (ou números de graus de liberdade) ao resíduo, que passou a ser 15 graus de liberdade.

\subsection{Métodos}

\subsubsection{Modelo Matemático}

O modelo matemático adotado para a análise conjunta foi o seguinte:

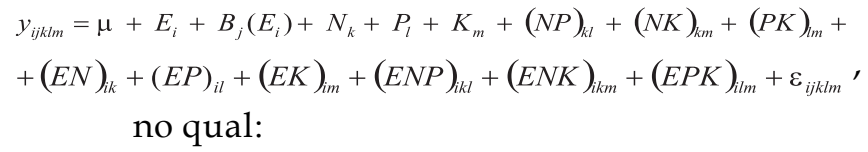

$\mathrm{y}_{\mathrm{ijklm}}$ é a produção de cana-planta, em t.ha-1, observada no experimento $i$, bloco $j$, para a $k l m$-ésima combinação dos fatores $\mathrm{N}, \mathrm{P}$ e $\mathrm{K}$ respectivamente;

e $m=1, \ldots, \mathrm{M}$;

$$
i=1, \ldots, \mathrm{I} ; j=1, \ldots, \mathrm{J} ; k=1, \ldots, \mathrm{K} ; l=1, \ldots, \mathrm{L}
$$

$\mu$ é a média geral; aleatório;

$E_{i}$ é o efeito do experimento i, considerado

$B_{j}\left(E_{i}\right)$ é o efeito do bloco j dentro do experimento i, considerado aleatório;

$N_{k}$ é o efeito do fator nitrogênio, considerado fixo;

$P_{l}$ é o efeito do fator fósforo, considerado fixo;

$K_{m}$ é o efeito do fator potássio, considerado fixo;

$(N P) k_{l}$ é o efeito da interação nitrogênio com fósforo, considerado fixo;

$(N P) k_{m}$ é o efeito da interação nitrogênio com potássio, considerado fixo;

$(P K)_{l m}$ é o efeito da interação fósforo com potássio, considerado fixo;

$(E N)_{i k}$ é o efeito da interação experimento com nitrogênio, considerado aleatório;

$(E P)_{i l}$ é o efeito da interação experimento com fósforo, considerado aleatório;
$(E K)_{i m}$ é o efeito da interação experimento com potássio, considerado aleatório;

$(E N P)_{i k l}$ é o efeito da interação experimento com $\mathrm{N}$ e com $\mathrm{P}$, considerado aleatório;

$(E N K)_{i k m}$ é o efeito da interação experimento com $\mathrm{N}$ e com $\mathrm{K}$, considerado aleatório;

$(E P K)_{i l m}$ é o efeito da interação experimento com $\mathrm{P}$ e com $\mathrm{K}$, considerado aleatório;

$\varepsilon_{i j k l m}$ é o erro aleatório, normal e independentemente distribuído, com média zero variância $\sigma 2$.

\subsubsection{Resíduo Específico}

O método proposto para contornar a heterocedasticidade das variâncias das interações é decompor as somas de quadrados de tratamentos em um conjunto de componentes ortogonais. As somas de quadrados das interações, também, são decompostas do mesmo modo, para se obter um resíduo específico, o qual servirá para testar cada um dos componentes de tratamentos com o componente obtido das interações dos tratamentos com experimentos.

Como os níveis dos fatores de tratamentos, para o conjunto de dados considerado são equidistantes, essa decomposição pode ser feita de modo simples, utilizando os coeficientes dos polinômios ortogonais.

O cálculo das somas de quadrados para a regressão linear e quadrática, para os efeitos principais, é obtido da seguinte forma:

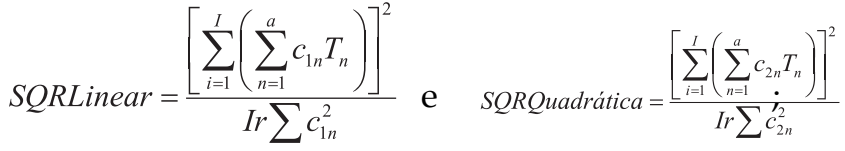

$$
\text { em que } \sum_{n=1}^{a} c_{1 n}=0, \sum_{\mathrm{n}=1}^{\mathrm{a}} \mathrm{c}_{2 \mathrm{n}}=0 \quad \text { e } \sum_{\mathrm{n}=1}^{\mathrm{a}} \mathrm{c}_{1 \mathrm{n}} c_{2 n}=0 \text { e }
$$

$c_{1 n}$ e $c_{2 n}$ são os coeficientes dos polinômios ortogonais de $1^{\circ}$ e $2^{\circ}$ graus respectivamente;

$T_{n}$ é o total das observações do n-ésimo nível do efeito considerado;

$r$ é o número de parcelas somadas para obter cada total $\left(T_{n}\right)$;

a é o número de níveis do efeito considerado;

I é o número de experimentos.

As somas de quadrados para as regressões linear e quadrática, para as interações são obtidas por:

SQRLinear $\times$ Exper. $=\frac{\sum_{i=1}^{I}\left(\sum_{n=1}^{a} c_{1 n} T_{n}\right)^{2}}{r \sum c_{1 n}^{2}}-$ SQRLinear 
e SQRQuadrática $\times$ Exper. $=\frac{\sum_{i=1}^{I}\left(\sum_{n=1}^{a} c_{2 n} T_{n}\right)^{2}}{r \sum c_{2 n}^{2}}-S Q R Q u a d r a ́ t i c a$

Um programa SAS foi elaborado para realizar as análises estatísticas propostas, utilizando o comando CONTRAST/ PROC GLM.

\section{RESULTADOS E DISCUSSÃO}

\subsection{Esperança dos quadrados médios}

Para se testar a hipótese de interesse, através do teste $\mathrm{F}$, é necessário estudar a natureza dos quadrados médios na análise de variância. Usando o modelo matemático adotado no item 2.2.1, foram consideradas as seguintes restrições:

(i) Para os efeitos fixos:

$\sum_{k} N_{k}=0 \sum_{;} P_{l}=0 \sum_{k, l}(N P)_{k l}=0 \sum_{i, m}(N K)_{k m}=0 \sum_{l, m}(P K)_{l m}=0$;.

(ii) Para os efeitos aleatórios:

$E_{i}, B_{j}\left(E_{i}\right),(E N)_{i k},(E P)_{i l},(E K)_{i m},(E N P)_{i k 1}$, $(\mathrm{ENK})_{\mathrm{ikm}},(\mathrm{EPK})_{\mathrm{ilm}}$ e $\boldsymbol{\varepsilon}_{i j k l m}$ são normais e independentemente distribuídos, com médias zero e variâncias $\sigma_{E}^{2}, \sigma_{B(E)}^{2}, \sigma_{E N}^{2}, \sigma_{E P}^{2}, \sigma_{E K}^{2}, \sigma_{E N P}^{2}, \sigma_{E N K}^{2}, \sigma_{E P K}^{2}, \sigma^{2}$ , respectivamente.

Além dessas restrições, considera-se que os efeitos aleatórios são não correlacionados.

Na tabela 1, estão apresentadas as esperanças matemáticas para os quadrados médios dos efeitos considerados no modelo, obtidas através do método dos momentos (Hicks, 1973).

A composição para aplicação do teste $\mathrm{F}$, considerando os resultados obtidos para as $\mathrm{E}(\mathrm{QM})$ foi realizada da seguinte forma:

(a) Para o efeito de Experimentos (E):

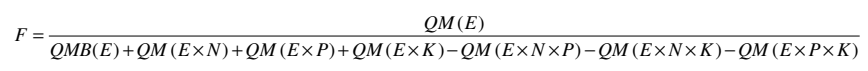

com (I-1) e $\mathrm{v}_{1}$ graus de liberdade, sendo $\mathrm{v}_{1}$, obtido pela fórmula de SATTERTHWAITE (1946).

(b) Para o efeito de doses de N:

$F=\frac{Q M(N)}{Q M(E x N)} \operatorname{com}(\mathrm{K}-1)$ e $(\mathrm{I}-1)(\mathrm{K}-1)$ graus de liberdade.

(c) Para o efeito de doses de P:

$F=\frac{Q M(P)}{\mathrm{QM}(\operatorname{ExP})} \operatorname{com}(\mathrm{L}-1)$ e $(\mathrm{I}-1)(\mathrm{L}-1)$ graus de liberdade. (d) Para o efeito de doses de K:

$F=\frac{Q M(K)}{\mathrm{QM}(\mathrm{ExK})} \operatorname{com}(\mathrm{M}-1)$ e $(\mathrm{I}-1)(\mathrm{M}-1)$ graus de liberdade.

(e) Para o efeito da interação NxP:

$F=\frac{Q M(N \times P)}{\mathrm{QM}(\mathrm{E} \times \mathrm{N} \times \mathrm{P})} \operatorname{com}(\mathrm{K}-1)(\mathrm{L}-1)$ e $(\mathrm{I}-1)(\mathrm{K}-1)(\mathrm{L}-1)$ graus de liberdade.

(f) Para o efeito da interação $N x K$ :

$F=\frac{Q M(N \times K)}{\mathrm{QM}(\mathrm{E} \times \mathrm{N} \times \mathrm{K})} \operatorname{com}(\mathrm{K}-1)(\mathrm{M}-1)$ e $(\mathrm{I}-1)(\mathrm{K}-1)(\mathrm{M}-1)$ graus de liberdade.

(g) Para o efeito da interação PxK:

$F=\frac{Q M(P \times K)}{\mathrm{QM}(\mathrm{E} \times P \times \mathrm{K})} \operatorname{com}(\mathrm{L}-1)(\mathrm{M}-1)$ e $(\mathrm{I}-1)(\mathrm{L}-1)(\mathrm{M}-1)$

graus de liberdade.

(h) Para o efeito da interação ExN:

$F=\frac{Q M(E \times N)}{Q M(E \times N \times P)+Q M(E \times N \times K)-Q M(\text { Resíduo Médio })}, \quad$ com $(\mathrm{I}-1)(\mathrm{K}-1)$ e $\mathrm{v}_{2}$ graus de liberdade, sendo $\mathrm{v}_{2}$, obtido pela fórmula de Satterthwaite (1946).

(i) Para o efeito da interação ExP:

$F=\frac{Q M(E \times P)}{Q M(E \times N \times P)+Q M(E \times P \times K)-Q M(\text { Resíduo Médio })}, \quad$ com $(\mathrm{I}-1)(\mathrm{L}-1)$ e $\mathrm{v}_{3}$ graus de liberdade, sendo $\mathrm{v}_{3}$, obtido pela fórmula de Satterthwaite (1946).

(j) Para o efeito da interação ExK:

$F=\frac{Q M(E \times K)}{Q M(E \times N \times K)+Q M(E \times P \times K)-Q M(\text { Resíduo Médio })}, \quad$ com (I-1)(M-1) e $\mathrm{v}_{4}$ graus de liberdade, sendo $\mathrm{v}_{4}$, obtido pela fórmula de Satterthwaite (1946).

(k) Para o efeito da interação ExNxP:

$F=\frac{Q M(E \times N \times P)}{Q M(\text { Resíduo Médio })} \quad, \operatorname{com}(\mathrm{I}-1)(\mathrm{K}-1)(\mathrm{L}-1)$ e I $(15)$ graus de liberdade.

(1) Para o efeito da interação ExNxK:

$F=\frac{Q M(E \times N \times K)}{Q M(\text { Resíduo Médio })}, \operatorname{com}(\mathrm{I}-1)(\mathrm{K}-1)(\mathrm{M}-1) \mathrm{e}$ I(15) graus de liberdade.

(m) Para o efeito da interação ExPxK:

$F=\frac{Q M(E \times P \times K)}{Q M(\text { Resíduo Médio })}, \operatorname{com}(\mathrm{I}-1)(\mathrm{L}-1)(\mathrm{M}-1) \mathrm{e}$

I(15) graus de liberdade.

3.1.1 Esperança dos quadrados médios para os componentes lineares e quadráticos de N, P e K

No caso dos experimentos de adubação, existe interesse em determinar a curva de produção da cultura em estudo, em função das doses crescentes 
aplicadas de N, P e K. Para isso, é usual decompor os efeitos de N, P e K em componentes lineares e quadráticos. A técnica utilizada é a dos polinômios ortogonais, e designa-se como $c_{1 \mathrm{k}}$ e $\mathrm{c}_{2 \mathrm{k}}$ os coeficientes dos polinômios linear e quadrático para o nitrogênio respectivamente.

A soma de quadrados para o efeito linear do nitrogênio é dada pela seguinte expressão:

$$
S Q\left(N^{\prime}\right)=\frac{1}{\operatorname{IJLM} \sum c_{1 k}^{2}}\left[\sum_{k} c_{1 k} y_{. . . .}\right]^{2}, \text { tal que } \sum_{k} c_{1 k}=0 .
$$

Assim,

$$
E\left[S Q\left(N^{\prime}\right)\right]=\frac{1}{I J L M \sum c_{1 k}^{2}} E\left[\sum_{k} c_{1 k} y_{. . . .}\right]^{2}
$$

em que,

$$
\begin{aligned}
& E\left[\sum_{k} c_{1 k} y_{. k . .}\right]^{2}=I^{2} J^{2} L^{2} M^{2}\left(\sum_{k} c_{1 k} N_{k}\right)^{2}+J^{2} L^{2} M^{2} \sum_{k} c_{1 k}^{2} I \sigma_{E N}^{2}+ \\
& +J^{2} M^{2} \sum_{k} c_{1 k}^{2} I L \sigma_{E N P}^{2}+J^{2} L^{2} \sum_{k} c_{1 k}^{2} I M \sigma_{E N K}^{2}+\sum_{k} c_{1 k}^{2} I J L M \sigma^{2} .
\end{aligned}
$$

Portanto,

$E\left[S Q\left(N^{\prime}\right)\right]=\sigma^{2}+J L \sigma_{E N K}^{2}+J M \sigma_{E N P}^{2}+J L M \sigma_{E N}^{2}+\frac{I J L M}{\sum c_{1 k}^{2}}\left(\sum_{k} c_{1 k} N_{k}\right)^{2}=E\left[Q M\left(N^{\prime}\right)\right]$ , (1) pois, a $S O\left(N^{\prime}\right)$ está associada a 1 número de grau de liberdade e $\sum c_{1 k} N_{k}=Y\left(N^{\prime}\right)$ é o valor teórico do contraste que corresponde ao efeito linear de $\mathrm{N}$.

Para o conjunto de dados em questão, o fator $\mathrm{N}$ tem três níveis, quantitativos, e os valores de $c_{1 \mathrm{k}}$ são referentes aos coeficientes do polinômio ortogonal de grau 1 , isto é, $c_{11}=-1, c_{12}=0$ e $c_{13}=1$. Logo,

$$
\begin{aligned}
& \left(\sum_{k} c_{1 k} N_{k}\right)^{2}=\left(c_{11} N_{1}+c_{12} N_{2}+c_{13} N_{3}\right)^{2}= \\
& =\left[(-1) N_{1}+(0) N_{2}+(1) N_{3}\right]^{2}=\left(N_{3}-N_{1}\right)^{2}=\left[Y\left(N^{\prime}\right)\right]^{2}
\end{aligned}
$$

Analogamente, utilizando $\mathrm{d}_{i l}$ como coeficientes do polinômio ortogonal para o efeito linear do fósforo e $\mathrm{e}_{l m}$ para o efeito linear do potássio, obtém-se:

$$
\begin{aligned}
& E\left[S Q\left(P^{\prime}\right)\right]=E\left[Q M\left(P^{\prime}\right)\right]=\sigma^{2}+J M \sigma_{E N P}^{2}+J K \sigma_{E P K}^{2}+J K M \sigma_{E P}^{2}+\frac{I J K M}{\sum d_{\| 1}^{2}}\left(\sum_{i} d_{11} P_{l}\right)^{2} \mathrm{e} \\
& E\left[S Q\left(K^{\prime}\right)\right]=E\left[Q M\left(K^{\prime}\right)\right]=\sigma^{2}+J L \sigma_{E N K}^{2}+J K \sigma_{F P K}^{2}+J K L \sigma_{E K}^{2}+\frac{I J K L}{\sum e_{l m}^{2}}\left(\sum_{m} e_{1 m} K_{m}\right)^{2},
\end{aligned}
$$

Sendo:

$\sum d_{1 l} P_{l}=Y\left(P^{\prime}\right)$ e $\sum e_{1 m} K_{m}=Y\left(K^{\prime}\right)$ os valores teóricos dos contrastes que correspondem aos efeitos lineares de, respectivamente, $\mathrm{P}$ e K.

Para obter as esperanças dos quadrados médios para os efeitos quadráticos de $\mathrm{N}, \mathrm{P}$ e K, basta substituir os coeficientes lineares $c_{1 k}, d_{11}$ e $e_{1 m}$ pelos coeficientes quadráticos $c_{2 k}, d_{21} \quad e \quad e_{2 m}$ respectivamente.

\subsubsection{Esperanças dos quadrados médios para} $\mathbf{N}^{\prime} \times \mathbf{P}^{\prime}, \mathbf{N}^{\prime} \times \mathbf{K}^{\prime}$ e $\mathbf{P}^{\prime} \times \mathbf{K}^{\prime}$.

Nos experimentos de adubação, com a finalidade de verificar se o efeito de um nutriente tem influência sobre o efeito do outro, existe interesse em estudar as interações de primeira ordem entre os nutrientes utilizados. As interações de interesse prático, segundo GOMEs (1990), são somente aquelas entre os efeitos lineares, isto é, $\mathrm{N}^{\prime} \times \mathrm{P}^{\prime}, \mathrm{N}^{\prime} \times \mathrm{K}^{\prime}$ e $\mathrm{P}^{\prime} \times \mathrm{K}^{\prime}$.

A soma de quadrados para $\mathrm{N}^{\prime} \mathrm{x}^{\prime} \mathrm{P}^{\prime}$ é dada pela seguinte expressão:

$$
\begin{aligned}
& S Q\left(N^{\prime} \times P^{\prime}\right)=\frac{1}{I J M \sum_{k l}\left(c_{1 k} d_{1 l}\right)^{2}}\left[\sum_{k l} c_{1 k} d_{1 l} y_{. k l}\right]^{2}, \\
& \text { tal que } \sum_{k, l} c_{1 k} d_{1 l}=0 \text { e } \sum_{k} c_{1 k}=\sum_{l} d_{1 l}=0 .
\end{aligned}
$$

Seguindo procedimento análogo à $\mathrm{N}^{\prime}, \mathrm{P}^{\prime}$ e $\mathrm{K}^{\prime}$, obtém-se que:

$$
\begin{aligned}
& E\left[S Q\left(N^{\prime} \times P^{\prime}\right)\right]=E\left[Q M\left(N^{\prime} \times P^{\prime}\right)\right]=\sigma^{2}+J M \sigma_{E N P}^{2}+\frac{I J M}{\sum_{k l}\left(c_{1 k} d_{l l}\right)^{2}}\left[\sum_{k l} c_{l k} d_{l l}(N P)_{k l}\right]^{2} ;, \\
& E\left[S Q\left(N^{\prime} \times K^{\prime}\right)\right]=E\left[Q M\left(N^{\prime} \times K^{\prime}\right)\right]=\sigma^{2}+J L \sigma_{E N K}^{2}+\frac{I J L}{\sum_{k m}\left(c_{1 k} e_{l m}\right)^{2}}\left[\sum_{k m} c_{1 k} e_{l m}(N K)_{k m}\right]^{2} ;, \\
& E\left[S Q\left(P^{\prime} \times K^{\prime}\right)\right]=E\left[Q M\left(P^{\prime} \times K^{\prime}\right)\right]=\sigma^{2}+J K \sigma_{E F K}^{2}+\frac{I J K}{\sum_{l m}\left(d_{11} e_{l m}\right)^{2}}\left[\sum_{l m} d_{l l} e_{l m}(P K)_{l m}\right]^{2},
\end{aligned}
$$

Sendo:

$\sum_{k l} c_{1 k} d_{1 l}(N P)_{k l}=Y\left(N^{\prime} \times P^{\prime}\right), \quad \sum_{k m} c_{1 k} e_{1 m}(N K)_{k m}=Y\left(N^{\prime} \times K^{\prime}\right)$ e $\sum d_{1 l} e_{1 m}(P K)_{l m}=Y\left(P^{\prime} \times K^{\prime}\right)$ os valores teóricos dos contrastes que correspondem aos efeitos de $\mathrm{N}^{\prime} \times \mathrm{P}^{\prime} \mathrm{x}$ , $\mathrm{N}^{\prime} \times \mathrm{K}^{\prime}$ e $\mathrm{P}^{\prime} \times \mathrm{K}^{\prime}$ respectivamente.

\subsubsection{Esperança dos quadrados médios para} as interações $\mathrm{E} \times \mathrm{N}^{\prime}$, E x P' e E x K'

A soma de quadrados para $\mathrm{E} \times \mathrm{N}^{\prime}$ é dada pela seguinte expressão:

$S Q\left(E \times N^{\prime}\right)=\frac{1}{J L M \sum_{k}^{2} c_{k}^{2}} \sum_{i}\left[\sum_{k} c_{k} y_{i k}\right]^{2}-S Q\left(N^{\prime}\right)$, tal que,. $\quad \sum_{k} c_{1 k}=0$

Aplicando-se esperança matemática, obtém-se

que, $E\left[S Q\left(E \times N^{\prime}\right)\right]=(I-1) \sigma^{2}+(I-1) J L \sigma_{E N K}^{2}+(I-1) J M \sigma_{E N P}^{2}+(I-1) J L M \sigma_{E N}^{2}$

e assim, $E\left[Q M\left(E \times N^{\prime}\right)\right]=\sigma^{2}+J L \sigma_{E N K}^{2}+J M \sigma_{E N P}^{2}+J L M \sigma_{E N}^{2}$.

$E\left[Q M\left(E \times P^{\prime}\right)\right]=\sigma^{2}+J M \sigma_{E N P}^{2}+J K \sigma_{E P K}^{2}+J K M \sigma_{E P}^{2}(2)$

Portanto, observando as equações (1) e (2), verifica-se que $\mathrm{QM}\left(\mathrm{EN}^{\prime}\right)$ é o resíduo específico para testar o efeito linear do nitrogênio $\left(\mathrm{N}^{\prime}\right)$.

Analogamente tem-se,

$E\left[Q M\left(E \times K^{\prime}\right)\right]=\sigma^{2}+J L \sigma_{E N K}^{2}+J K \sigma_{E P K}^{2}+J K L \sigma_{E K}^{2} \quad \mathrm{e}$ 
$S Q\left(E \times N^{\prime} \times P^{\prime}\right)=\frac{1}{J M \sum_{k l}\left(c_{1 k} d_{1 l}\right)^{2}} \sum_{i}\left(\sum_{k l} c_{1 k} d_{1 l} y_{i . k l .}\right)^{2}-S Q\left(N^{\prime} \times P^{\prime}\right)$, que correspondem, respectivamente, aos resíduos específicos para testar os efeitos lineares do fósforo $\left(\mathrm{P}^{\prime}\right)$ e do potássio $\left(\mathrm{K}^{\prime}\right)$.

\subsubsection{Esperança dos quadrados médios para $\mathrm{E} \times \mathrm{N}^{\prime}$ $x \mathbf{P}^{\prime}, \mathrm{E} \times \mathrm{N}^{\prime} \times \mathrm{K}^{\prime}$ e $\mathrm{E} \times \mathbf{P}^{\prime} \times \mathrm{K}^{\prime}$}

A soma de quadrados para $E N^{\prime} \mathrm{P}^{\prime}$ é dada pela seguinte expressão:

$S Q\left(E \times N^{\prime} \times P^{\prime}\right)=\frac{1}{J M \sum_{k l}\left(c_{1 k} d_{1 l}\right)^{2}} \sum_{i}\left(\sum_{k l} c_{1 k} d_{1 l} y_{i . k l .}\right)^{2}-S Q\left(N^{\prime} \times P^{\prime}\right)$,

tal que, $\sum_{k, l} c_{1 k} d_{1 l}=0$ e $\sum_{k} c_{1 k}=\sum_{l} d_{1 l}=0$.
Aplicando-se esperança matemática, obtém-se que, $E\left[S Q\left(E \times N^{\prime} \times P^{\prime}\right)\right]=(I-1) \sigma^{2}+(I-1) J M \sigma_{E N P}^{2}$. e assim

$E\left[Q M\left(E \times N^{\prime} \times K^{\prime}\right)\right]=\sigma^{2}+J L \sigma_{E N K}^{2}$.

Analogamente tem-se,

$$
\begin{aligned}
& E\left[Q M\left(E \times N^{\prime} \times K^{\prime}\right)\right]=\sigma^{2}+J L \sigma_{E N K}^{2} \mathrm{e} \\
& E\left[Q M\left(E \times P^{\prime} \times K^{\prime}\right)\right]=\sigma^{2}+J K \sigma_{E P K}^{2} .
\end{aligned}
$$

que correspondem, respectivamente, aos resíduos específicos para testar as interações

$$
\mathrm{N}^{\prime} \times \mathrm{P}^{\prime}, \mathrm{N}^{\prime} \times \mathrm{K}^{\prime} \text { e } \mathrm{P}^{\prime} \times \mathrm{K}^{\prime} \text {. }
$$

\begin{tabular}{|c|c|c|}
\hline Causas de Variação & GL & $\mathrm{E}(\mathrm{QM})$ \\
\hline E & $\mathrm{I}-1$ & $\begin{array}{l}\sigma^{2}+J K \sigma_{E P K}^{2}+J L \sigma_{E N K}^{2}+J M \sigma_{E N P}^{2}+J K L \sigma_{E K}^{2}+ \\
+J K M \sigma_{E P}^{2}+J L M \sigma_{E N}^{2}+K L M \sigma_{B(E)}^{2}+J K L M \sigma_{E}^{2}\end{array}$ \\
\hline $\mathrm{B}(\mathrm{E})$ & $\mathrm{I}(\mathrm{J}-1)$ & $\sigma^{2}+K L M \sigma_{B(E)}^{2}$ \\
\hline $\mathrm{N}$ & $\mathrm{K}-1$ & $\sigma^{2}+J L \sigma_{E N K}^{2}+J M \sigma_{E N P}^{2}+J L M \sigma_{E N}^{2}+I J L M \phi_{N}$ \\
\hline $\mathrm{P}$ & L-1 & $\sigma^{2}+J M \sigma_{E N P}^{2}+J K \sigma_{E P K}^{2}+J K M \sigma_{E P}^{2}+I J K M \phi_{P}$ \\
\hline K & M-1 & $\sigma^{2}+J L \sigma_{E N K}^{2}+J K \sigma_{E P K}^{2}+J K L \sigma_{E K}^{2}+I J K L \phi_{K}$ \\
\hline $\mathrm{NxP}$ & $(\mathrm{K}-1)(\mathrm{L}-1)$ & $\sigma^{2}+J M \sigma_{E N P}^{2}+I J M \phi_{N P}$ \\
\hline $\mathrm{NxK}$ & $(\mathrm{K}-1)(\mathrm{M}-1)$ & $\sigma^{2}+J L \sigma_{E N K}^{2}+I J L \phi_{N K}$ \\
\hline PxK & $(\mathrm{L}-1)(\mathrm{M}-1)$ & $\sigma^{2}+J K \sigma_{E P K}^{2}+I J K \phi_{P K}$ \\
\hline ExN & $(\mathrm{I}-1)(\mathrm{K}-1)$ & $\sigma^{2}+J L \sigma_{E N K}^{2}+J M \sigma_{E N P}^{2}+J L M \sigma_{E N}^{2}$ \\
\hline ExP & $(\mathrm{I}-1)(\mathrm{L}-1)$ & $\sigma^{2}+J K \sigma_{E P K}^{2}+J M \sigma_{E N P}^{2}+J K M \sigma_{E P}^{2}$ \\
\hline ExK & $(\mathrm{I}-1)(\mathrm{M}-1)$ & $\sigma^{2}+J K \sigma_{E P K}^{2}+J L \sigma_{E N K}^{2}+J K L \sigma_{E K}^{2}$ \\
\hline $\mathrm{ExNxP}$ & $(\mathrm{I}-1)(\mathrm{K}-1)(\mathrm{L}-1)$ & $\sigma^{2}+J M \sigma_{E N P}^{2}$ \\
\hline $\mathrm{ExN} \times \mathrm{K}$ & $(\mathrm{I}-1)(\mathrm{K}-1)(\mathrm{M}-1)$ & $\sigma^{2}+J L \sigma_{E N K}^{2}$ \\
\hline ExPxK & $(\mathrm{I}-1)((\mathrm{L}-1)(\mathrm{M}-1)$ & $\sigma^{2}+J K \sigma_{E P K}^{2}$ \\
\hline Resíduo Médio & $\mathrm{I}(15)$ & $\sigma^{2}$ \\
\hline
\end{tabular}

Tabela 1. Esquema da análise da variância, com as esperanças matemáticas dos quadrados médios, para o modelo considerado na análise conjunta.

$\phi_{N}=\frac{1}{K-1} \sum_{k} N_{k}^{2} \quad \phi_{P}=\frac{1}{L-1} \sum_{l} P_{l}^{2}$

$\phi_{K}=\frac{1}{M-1} \sum_{m} K_{m}^{2}$

$\phi_{N P}=\frac{1}{(K-1)(L-1)} \sum_{k l}(N P)_{k l}^{2} \quad \phi_{N K}=\frac{1}{(K-1)(M-1)} \sum_{k m}(N K)_{k m}^{2} \quad \phi_{P K}=\frac{1}{(L-1)(M-1)} \sum_{l m}(P K)_{l m}^{2}$ 


\subsection{Verificação da homocedasticidade entre experimentos}

O teste utilizado para verificar a homogeneidade das variâncias residuais, entre experimentos, foi o teste de Bartlett, sendo essa hipótese rejeitada, a de $5 \%$ de probabilidade. $\mathrm{Na}$ tabela 2, observam-se os quadrados médios dos resíduos para os experimentos.

Tabela 2. Quadrados médios dos resíduos para os experimentos

\begin{tabular}{lcc}
\hline Experimentos (i) & GL & QMResíduo (i) \\
\hline 1 & 15 & 118,41 \\
2 & 15 & 61,95 \\
3 & 15 & 104,58 \\
4 & 15 & 117,82 \\
5 & 15 & 280,02 \\
6 & 15 & 470,91 \\
7 & 15 & 231,96 \\
8 & 15 & 302,49 \\
9 & 15 & 608,83 \\
10 & 15 & 250,25 \\
11 & 15 & 500,36 \\
\hline
\end{tabular}

\subsection{Análise de variância conjunta}

O teste $\mathrm{F}$, aplicado na análise de variância, tem os valores obtidos de acordo com os resultados das respectivas esperanças dos quadrados médios, apresentados em 3.1, cujos resultados são mostrados na tabela 3.

Pela tabela 3, verifica-se que todas as interações envolvendo o fator experimentos não foram significativas, isto é, os efeitos de N, P e K foram semelhantes nos diferentes experimentos (locais). As interações $\mathrm{N}^{\prime} \mathrm{P}^{\prime}, \mathrm{N}^{\prime} \mathrm{K}^{\prime}$ e $\mathrm{P}^{\prime} \mathrm{K}^{\prime}$ não foram significativas, posto que o efeito de um fertilizante não interfere no efeito do outro, em média. Observou-se, também, que os efeitos médios para $\mathrm{N}$ e $\mathrm{K}$ foram significativos. Assim, realizou-se a decomposição dos dois graus de liberdade associados à soma de quadrados de cada um deles, ou seja, um referente ao efeito linear e outro, ao efeito quadrático do fertilizante. $\mathrm{O}$ teste $\mathrm{F}$, para os efeitos linear e quadrático de $\mathrm{N}$ e $\mathrm{K}$, tem como denominadores EN e EK respectivamente. Neste trabalho, o interesse está em decompor as somas de quadrados das interações EN e EK para a obtenção dos resíduos específicos, para se testar cada um desses efeitos, devido à heterocedasticidade presente nas variâncias residuais entre experimentos. Os resultados são apresentados na tabela 4 .

Tabela 3. Resultados obtidos da análise de variância conjunta para os dados de rendimentos de cana de açúcar.

\begin{tabular}{|c|c|c|c|c|c|}
\hline Causas de Variação & G.L. & SQ & $\mathrm{QM}$ & $\mathrm{F}$ & $\operatorname{Pr}>F$ \\
\hline Experimentos (E) & 10 & 1185325,80 & 118532,58 & 157,94 & $<0,0001$ \\
\hline Blocos dentro $\mathrm{E}$ & 22 & 10989,36 & 499,52 & - & - \\
\hline $\mathrm{N}$ & 2 & 3931,39 & 1965,70 & 5,02 & 0,0214 \\
\hline $\mathrm{P}$ & 2 & 643,38 & 321,69 & 1,84 & 0,1929 \\
\hline K & 2 & 12741,20 & 6370,60 & 12,13 & 0,0007 \\
\hline $\mathrm{N}^{\prime} x \mathrm{P}^{\prime}$ & 1 & 39,82 & 39,82 & 0,17 & 0,6888 \\
\hline $\mathrm{N}^{\prime} x \mathrm{~K}^{\prime}$ & 1 & 29,69 & 29,69 & 0,09 & 0,7703 \\
\hline $\mathrm{P}^{\prime} \times \mathrm{K}^{\prime}$ & 1 & 30,07 & 30,07 & 0,12 & 0,7362 \\
\hline ExN & $20(15)$ & 7824,07 & 391,20 & 1,30 & 0,4137 \\
\hline ExP & $20(15)$ & 3495,72 & 174,79 & 0,85 & 0,6482 \\
\hline ExK & $20(15)$ & 10505,16 & 525,26 & 1,73 & 0,2837 \\
\hline ExN'xP' & 10 & 2390,66 & 239,07 & 0,86 & 0,5725 \\
\hline $\mathrm{ExN}^{\prime} x \mathrm{~K}^{\prime}$ & 10 & 3380,58 & 338,06 & 1,22 & 0,2852 \\
\hline $\operatorname{ExP}^{\prime} x K^{\prime}$ & 10 & 2424,8 & 242,48 & 0,88 & 0,5539 \\
\hline Resíduo Médio & 165 (119) & 45713,82 & 277,05 & - & - \\
\hline Total & 296 & 1289465,59 & - & - & - \\
\hline
\end{tabular}

Nota: Os números entre parênteses são referentes ao número de graus de liberdade ajustados pelo método de Cochran (1954) e considerados no cálculo da Pr>F. 
Na tabela 5, constata-se a aplicação do teste $\mathrm{F}$, sem utilizar o resíduo específico. Confrontando as tabelas 4 e 5, observa-se que o denominador na composição do teste $\mathrm{F}$, para $\mathrm{N}^{\prime}$ e $\mathrm{N}^{\prime \prime}$ é o $\mathrm{QM}(\mathrm{EN})$, que representa o valor médio entre $\mathrm{QM}\left(\mathrm{EN}^{\prime}\right)$ e $\mathrm{QM}\left(\mathrm{EN}^{\prime \prime}\right)$. Se estes valores são relativamente diferentes, o denominador com o QM(EN) não é o mais adequado.

Tabela 4. Resultados obtidos na análise de variância aplicando o resíduo específico

\begin{tabular}{|c|c|c|c|c|c|}
\hline Causas de Variação & GL & SQ & $\mathrm{QM}$ & $\mathrm{F}$ & $\operatorname{Pr}>F$ \\
\hline $\mathrm{N}^{\prime}$ & 1 & 3703,28 & 3703,28 & 14,97 & 0,0031 \\
\hline $\mathrm{N}^{\prime \prime}$ & 1 & 228,11 & 228,11 & 0,43 & 0,5269 \\
\hline $\mathrm{K}^{\prime}$ & 1 & 12126,01 & 12126,01 & 13,85 & 0,0040 \\
\hline $\mathrm{K}^{\prime \prime}$ & 1 & 615,19 & 615,19 & 3,51 & 0,0905 \\
\hline $\mathrm{ExN}^{\prime}$ & 10 & 2473,51 & 247,35 & - & - \\
\hline ExN" & 10 & 5350,56 & 535,06 & - & - \\
\hline ExK' & 10 & 8754,29 & 875,43 & - & - \\
\hline ExK" & 10 & 1750,87 & 175,09 & - & - \\
\hline
\end{tabular}

Tabela 5. Resultados obtidos na análise de variância sem aplicar o resíduo específico.

\begin{tabular}{lrrrrr}
\hline Causas de Variação & GL & \multicolumn{1}{c}{ SQ } & QM & \multicolumn{1}{c}{ F } & $\operatorname{Pr}>$ F \\
\hline $\mathrm{N}^{\prime}$ & 1 & 3703,28 & 3703,28 & 9,47 & 0,0077 \\
$\mathrm{~N}^{\prime \prime}$ & 1 & 228,11 & 228,11 & 0,58 & 0,4581 \\
$\mathrm{~K}^{\prime}$ & 1 & 12126,01 & 12126,01 & 23,09 & 0,0002 \\
$\mathrm{~K}^{\prime \prime}$ & 1 & 615,19 & 615,19 & 1,17 & 0,2965 \\
ExN & $20(15)$ & 7824,07 & 391,20 & - & - \\
ExK & $20(15)$ & 10505,16 & 525,26 & - & - \\
\hline
\end{tabular}

\section{CONCLUSÕES}

1. As interações $\mathrm{E} \times \mathrm{N}^{\prime}, \mathrm{E} \times \mathrm{P}^{\prime}, \mathrm{E} \times \mathrm{K}^{\prime}, \mathrm{E} \times \mathrm{N}^{\prime \prime}$, $E \times \mathrm{P}^{\prime \prime}$ e $\mathrm{E} \times \mathrm{K}^{\prime \prime}$ são os resíduos específicos para se testar os efeitos de $\mathrm{N}^{\prime}, \mathrm{P}^{\prime}, \mathrm{K}^{\prime}, \mathrm{N}^{\prime \prime}, \mathrm{P}^{\prime \prime}$ e $\mathrm{K}^{\prime \prime}$ respectivamente. Da mesma forma que as interações $E \times N^{\prime} P^{\prime}, E \times N^{\prime} K^{\prime}$ e $E \times P^{\prime} K^{\prime}$ são os resíduos específicos para se testar os efeitos de $\mathrm{N}^{\prime} \times \mathrm{P}^{\prime}, \mathrm{N}^{\prime} \mathrm{x}$ $\mathrm{K}^{\prime}$ e $\mathrm{P}^{\prime} \times \mathrm{K}^{\prime}$ respectivamente.

2. A técnica do resíduo específico, aplicada nos dados experimentais apresentados, é alternativa adequada, de análise de dados de grupo de experimentos, na presença de heterocedasticidade entre experimentos.

\section{REFERÊNCIAS}

BANZATTO, D.A.; KRONKA, S.N. Experimentação Agrícola. Jaboticabal: FUNEP-UNESP, 1989. 247p.

COCHRAN, W.G. The combination of estimates from different experiments. Biometrics, Baltimore, v.10, n.1, p.101-129, 1954.

COCHRAN, W.G.; COX, G.M. Experimental Designs. 2.ed. New York: John Wiley and Sons, 1957. 611p.
GOMES, F.P. Curso de Estatística Experimental. 13.ed. Piracicaba: Nobel, 1990. 467p.

HICKS, C.R. Fundamental Concepts in the Design of Experiments. 2.ed. New York: Holt, Rinehart and Winston, 1973. 349p.

KEMPTHORNE, O. The design and analysis of experiments. New York: John Wiley and Sons, 1952. 631p.

MALAVOLTA, E. et al. A diagnose foliar na cana-de-açucar IV. Resultados de 40 ensaios fatoriais NPK 333, primeiro corte no estado de São Paulo. ESALQ-USP, Piracicaba, 1963. 47p.

NOGUEIRA, M.C.S. Orthogonal Contrasts: Definitions and Concepts. Scientia Agricola, Piracicaba, v. 61, n. 1, p. 118-124, 2004.

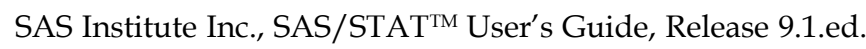
Cary, NC: SAS Institute Inc., 2006.

SATTERTHWAITE, F.E. An approximate distribution of estimates of variance components. Biometrics Bulletin, Washington, v.2, p.110-114, 1946.

YATES, F. The design and analysis of factorial experiments. Imperial Bureau of Soil Science, Harpenden, v.35, 1937.

YATES, F.; COCHRAN, W.G. The analysis of groups of experiments. Journal of Agricultural Science, Harpenden, v.28, p.556-580, 1938. 